\title{
Thymoma B3 \& Myasthenia Gravis in Adolescent: Case Report
}

\section{Basil Khadim ${ }^{1 *}$, Hiba Tawfiq $\mathrm{F}^{2}$}

${ }^{1}$ Consultant Subspecialty Pediatric Heamato-Oncology, Hiwa Teaching Hospital, Sulemania, Iraq

${ }^{2}$ High Diploma Student, Pediatric Oncology, Hiwa Teaching Hospital, Sulimania, Iraq

\begin{abstract}
A thymoma is typically a slow growing tumor, it cells look similar to the normal cell of the thymus and it usually does not spread beyond the thymus. People with thymoma, however, often have other disease as well the most common myasthenia gravis, a disease that weaken the muscles but other includes polymyositis, lupus erythromatosus, rheumatoid arthritis, thyroiditis and hypogammaglobulinemia. Thymoma arises from thymic epithelium and could be malignant or benign tumor. Thymoma is rare, accounting for about $0.2 \%$ to $0.5 \%$ of all cancers. Approximately $90 \%$ of tumor of thymus is thymoma; the remaining $10 \%$ are thymic carcinoma, carcinoid tumor or lymphoma. Thymoma is one of the rare pediatric tumors. Only $2 \%$ of all pediatric mediastinal tumors are thymoma. Less than 35 cases in pediatric have been reported. Thymoma nay be diagnosed because of other neuromuscular disorders mostly myasthenia gravis. However, this correlation is less common in pediatric thymoma. When occurred the tumor usually more aggressive, with more mortality. We reported a case of myasthenia gravis associated with thymoma in 16 yrs. old male.
\end{abstract}

Keywords: Mediastinal mass; Thymoma; Myasthenia gravis

Abbreviations: CXR: Chest X-Ray; CT: Computed Tomography; MG: Myasthenia Gravis; PET: Positron Emission Tomography; LT: Left; US: Ultra Sound; G-CSF: Granulocyte-Colony Stimulating Factor; LN: Lymph Nodes

\section{Case Report}

We presented A 16 years old male presented with history recurrent attacks of Shortness of breath since 3 month ago which was treated symptomatically. Since 1 month age he was sever dyspnic and admitted to hospital he was cyanosis, after established his respiratory attacks, CXR (Chest X-Ray) reveal multiple mediastinal opacification (Figure 1). CT (Computed Tomography) chest done reveal multiple mediastinal masses $(80 \times 70 \times 50) \mathrm{mm}$ that compress the great vessels and heart with mild LT (Left) pleural thickening (Figure 2). His condition associated with ptosis (Figure 3) of both eyelid (falling of eyelid), CT brain was normal. LT thoracotomy with incision biopsy and histopathology reveal B3 thymoma (atypical thymoma), staging not applicable (incision

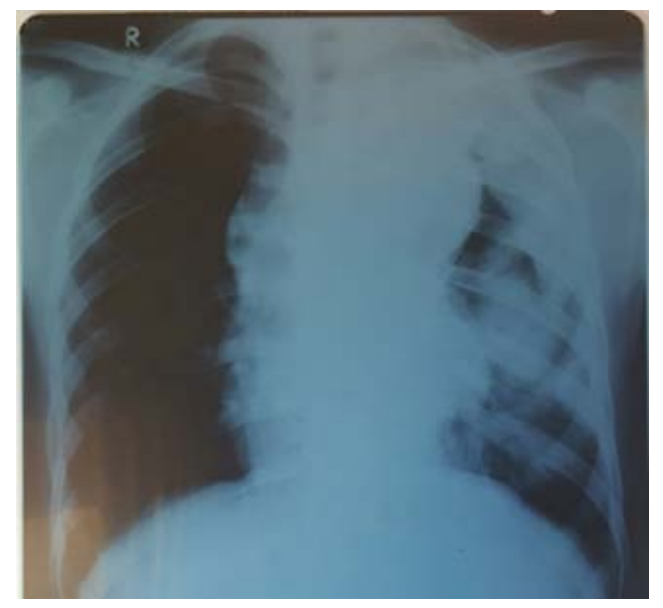

Figure 1: Respiratory attacks, CXR reveal multiple mediastinal opacification.

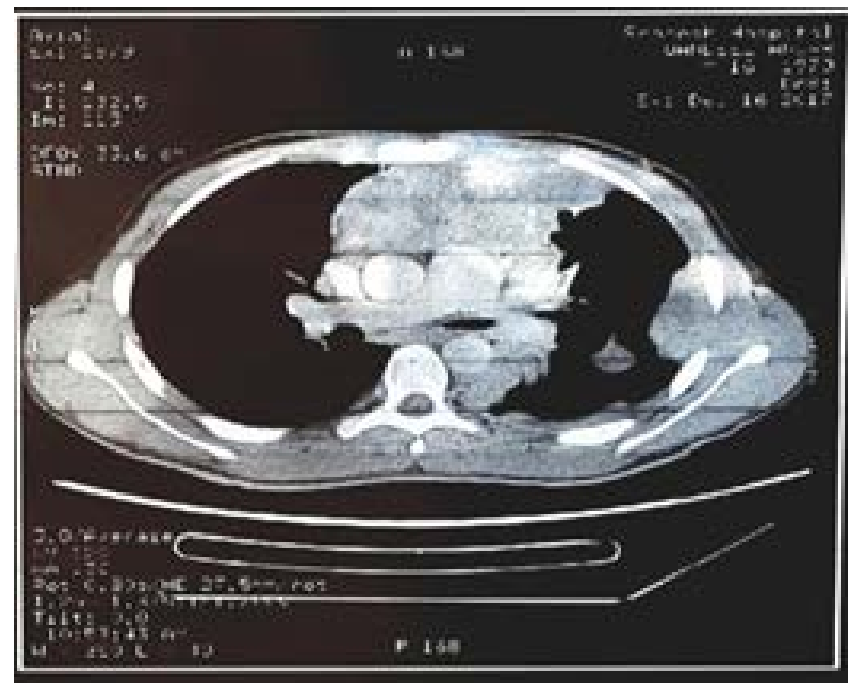

Figure 2: Vessels and heart with mild LT side pleural thickening.

biopsy). Immunohistochemistry was performed with following result for indicated antibody with positive control: strongly positive of cell membrane was (AE1/AE2), positive (CD68), negative (CD20, CD3, CD79a, CD10, CD30, CD117, TdT) (BCL2, BCL6). The patient referred

*Corresponding author: Basil Khadim, Consultant Subspecialty Pediatric Heamato-Oncology, Hiwa Teaching Hospital, Sulemania, Iraq, Tel: +9647705078475; E-mail: basilonc@yahoo.com

Received August 18, 2018; Accepted September 27, 2018; Published October 05, 2018

Citation: Khadim B, Hiba Tawfiq F (2018) Thymoma B3 \& Myasthenia Gravis in Adolescent: Case Report. J Oncol Med \& Pract 3: 122. doi:10.4172/25763857.1000122

Copyright: $\odot 2018$ Khadim B, et al. This is an open-access article distributed under the terms of the Creative Commons Attribution License, which permits unrestricted use, distribution, and reproduction in any medium, provided the original author and source are credited. 


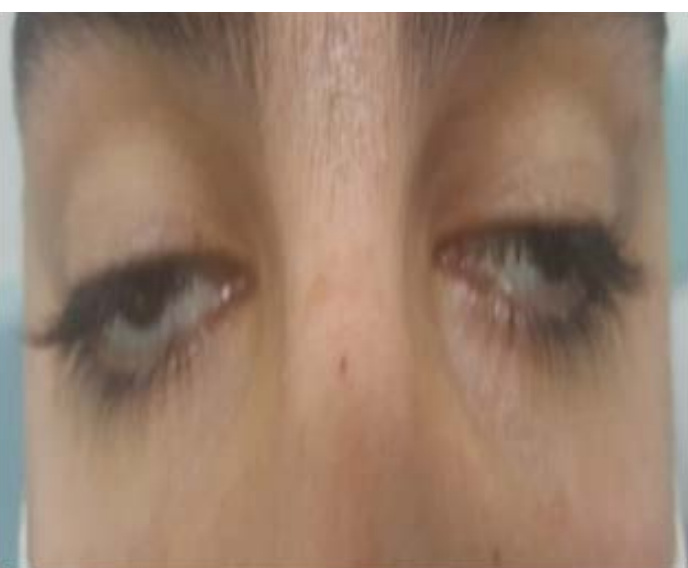

Figure 3: Patient ptosis.

to oncology department in Hiwa hospital to consultant Dr. Basil Khadim (Pediatric Heamato-Oncology Subspecialty) for complete clinical evaluation and management.

\section{Physical Examination}

On physical examination there was alert not dyspnic or pale, ptosis eyelid, respiratory rate $24 / \mathrm{min}$ and auscultation of the chest was harsh vesicular breathing. Other physical examination finding were normal laboratory finding were normal including (complete blood count + blood film, renal function, liver function, electrolyte, thyroid function test, tumor markers (beta-human chorionic gonadotrophin and alphafetoprotein), viral screen, acetylcholine esterase inhibitor $\mathrm{AB}$, titin \& Ryanodine Receptor (RYR) AB.

Regarding image study include: CXR multiple medistinal opacification, abdomen US was normal, Echocardiography was unremarkable, Nerve conduction study suggestive of MG (Myasthenia Gravis) based on trapezius muscle and needle Electromyography data reveal myopathic unit which seen in MG, CT chest, abdomen and pelvic post incisional biopsy reveal (diffuse enlargement of the anterior mediastinal LNs (Lymph Nodes) that are matted together and form an in homogenous mass measuring about $(65 \times 26) \mathrm{mm}$ with spot of calcification or atelectasis (residual thymoma) with borderline spleenomegaly otherwise is normal), PET (Positron Emission Tomography) scan for re-staging.

Within 1 week the patient's progress extra ocular weakness to slurred speech and dysphagia regarding his MG we started with IV/ IG $(1 \mathrm{G} / \mathrm{GK} / 12 \mathrm{~h})$ and put him on long life systemic steroid with ACHE inhibitor (pyridostigmine bromide $60 \mathrm{mg}$ ). Regarding his thymoma we started chemotherapy and planned (3-4) cycle of adriamycine, cisplatin, cyclophosphamide every 21 days supported by G-CSF (Granulocytecolony Stimulating Factor)) and mediastinal irradiation.

Unfortunately the patient immediately after the first cycle of chemotherapy developed severe respiratory failure for which he is admitted to the intensive care unit and intubated; he died after few days from admission to the intensive care unit.

\section{Discussion}

Approximately $30 \%$ of the patients with thymoma are presented with space occupying sign and symptoms on the nearby tissues as dysphagia, cough, chest pain, or superior vena cava syndrome caused by growing mass. Other $30 \%$ may present with autoimmune disease as pure red cell aplasia, inflammatory bowel disease, immunodeficiency, hemolytic anemia, and the most common MG [1]. This correlation were much less commonly recognized in pediatric, possibly because of the rarity of thymoma in pediatric. Other $30 \%$ of the patients are asymptomatic may diagnosed radio-logically performed for unrelated disease. Primary thymus lesion (such as thymus cyst, thymolipoma and thymus hyperplasia) only represent $2.5 \%$ of mediastinal tumor [2-4]. The incidence of myasthenia gravis in the general population is thought to be $0.5 / 100.000$ to $3 / 100.000$ and approximately $1 \%$ of all patient with MG are children [3]. Thymoma arisen from the thymic epithelium and microscopically could be type A with an oval shape, type B with epithelioid shape, or type $A B$ with combination of the two shapes. Our patient was complain from progressive weakness that involving the respiratory muscle and causing progressive respiratory failure. CXR multiple medistinal opascification, CT scan showed diffuse enlargement of the anterior mediastinal LNs that are matted together and form an in homogenous mass measuring about $(65 \times 26) \mathrm{mm}$ with spot of calcification or atelectasis (residual thymoma) with borderline spleenomegaly otherwise is normal, CT scan is important as Chest $\mathrm{X}$-ray may be normal in patient with thymoma, it is also important for staging [5]. Antibodies to the acetylcholine receptor was positive in our patient. The World Health Organization (WHO) recently developed a classification system (Table 1) [6].

\section{The Masaoka staging system}

IA: Completely encapsulated.

IIA: Microscopic invasion through the capsule into surrounding fatty tissue.

IIB: Macroscopic invasion into capsule.

III: Macroscopic invasion into adjacent organs.

IVA: Pleural or pericardial implants.

IVB: Lymphogenous or hematogenous metastasis to distant (extra thoracic) sites.

Surgery is the most important part of treatment with complete surgical resection being the treatment of choice for both local control and autoimmune manifestations $[7,8]$. MG in our patient was treated by an ant cholinesterase drugs and prednisone. Thymectomy has been performed on patient who was refractory to drug therapy [7]. Our patients was also treated by chemotherapy and irradiation. Respiratory failure remain an important cause of mortality $[9,10]$. Other causes of death include infection, side effects of treatment. The prognosis in pediatric still unrecognized because of the rarity of thymoma during childhood [11,12].

In summary, the association of MG and thymoma, although rare, dose occurs in the pediatric population. We report a 16 yrs old male with this association.

\begin{tabular}{|l|l|}
\hline \multicolumn{1}{|c|}{ Type } & \multicolumn{1}{c|}{ Histologist Description } \\
\hline A & Medullary Thymoma. \\
\hline AB & Mixed Thymoma. \\
\hline B1 & Predominantly Cortical Thymoma. \\
\hline B2 & Cortical Thymoma. \\
\hline B3 & Well- differentiated Thymic carcinoma. \\
\hline C & Thymic Carcinoma. \\
\hline
\end{tabular}

Table 1: WHO Classification. 


\section{Conclusion}

There are known and established relationships between thymoma and myasthenia gravis although thymomatous myasthenia gravis tend to have a difficult clinical course and poor prognosis, early and aggressive treatment along with multidisciplinary management may improve the outcome of this patient.

\section{Consent}

Written informed consent was obtained from both patient for population of this case report and any accompanying image.

\section{Declarations}

\section{Acknowledgments} report.

We gratefully acknowledge both patients for allowing us to publish their case

\section{Authors' contributions}

Consultant DR. Basil have involved in clinical diagnostic evaluations and management.

\section{References}

1. King RM, Telander RL, Smithson WA (1982) Primary mediastinal tumors in children. J Pediatr Surg 5: 512-520.
2. Cohen AJ, Thompson L, Edwards FH (1991) Primary cysts and tumors of the mediastinum. Ann Thorac Surg 51: 378-384.

3. Souadjian JV, Enriquez P, Silverstein MN, Pepin JM (1974) The spectrum of diseases associated with thymoma. Coincidence or syndrome? Arch Intern Med 134: 374-379.

4. Simpson JA (1974) Myasthenia gravis and myasthenia syndromes. Disorders of Voluntary Muscle. Edinburgh, London, New York: Churchill Livingston, pp. 665-666.

5. Bertelse S, Malmstrom J, Heerfordt J (1975) Tumors of the thymus regionSymptomatology, diagnosis, treatment and prognosis. Thorax 30: 19-25.

6. Masaoka A, Monden Y, Nakahara K (1981) Following study of thymoma with special reference to their clinical stages. Cancer 48: 2485-2492.

7. Haniuda M, Miyazawa M, Yoshida (1996) Is postoperative radiotherapy for thymoma effective? Ann Surg 224: 219-224.

8. Fornasiero A, Daniele O, Ghiotto C (1991) Chemotherapy for invasive thymoma. A 13-year experience. Cancer 68: 30-33.

9. Legg MA, Brady WJ (1968) Pathology and clinical behavior of thymomas: A survey of 51 cases. Cancer 18: 1131-1144.

10. Namba T, Brunner NG, Grob D (1978) Myasthenia gravis in patients with thymoma with particular reference to onset after thymectomy. Medicine 57: 411-434.

11. Rodriguez M, Gomez MR, Howard FM, Taylor WF (1983) Myasthenia gravis in children: Long-term follow-up. Ann Neural 13: 504-510.

12. Cavanaugh NPC (1980) The role of thymectomy in childhood myasthenia Develop Med Child Neural 22: 668-667. 